\title{
NONLINEAR APPROXIMATION. II CURVATURE IN MINKOWSKI GEOMETRY AND LOCAL UNIQUENESS
}

\author{
BY \\ JOHN R. RICE( $\left.{ }^{1}\right)$
}

1. Introduction. This paper continues the development started in [8] of a geometric theory of nonlinear approximation. The first part develops the geometry of finite-dimensional Minkowski spaces with regard to tangents and normals (\$2) and curvature ( $\$ 3)$ of submanifolds. The second part concerns metric projections on submanifolds of such spaces. Several basic, but known or easy, results are stated ( $\$ 4)$ in addition to the principal result of this paper, a local uniqueness theorem (\$5). It states: Let $\mathscr{F}$ be a closed submanifold of a smooth Minkowski space and let $\mathscr{F}$ have curvature bounded on compact subsets of $\mathscr{F}$. Then there is a neighborhood $\mathscr{F}_{u}$ of $\mathscr{F}$ such that every point in $\mathscr{F}_{u}$ has a unique projection onto $\mathscr{F}$. Quantitative results are also given. The next part (\$6) presents a relation between the topological nature of $\mathscr{F}$ and that of the set of points which do not have a unique projection onto $\mathscr{F}$. The final part ( $\$ 7)$ contains remarks on the direction of study for nonmanifold problems. These problems include the most interesting ones of nonlinear approximation.

2. Results concerning tangents and normals. We consider a Banach space $B^{m}$ of dimension $m$. The norm is denoted by $\|\quad\|$ and the ball with center $b_{0}$ and radius $\rho$ is

$$
S\left(b_{0}, \rho\right)=\left\{b \mid\left\|b-b_{0}\right\|<\rho\right\} .
$$

Its boundary is the sphere $\partial S\left(b_{0}, \rho\right)$ and its closure is the closed ball $\bar{S}\left(b_{0}, \rho\right)$. If $b_{1}$ and $b_{2}$ are distinct points then the set of points of the form $(1-\lambda) b_{1}+\lambda b_{2}$ form for

$$
\begin{array}{cl}
-\infty & <\lambda<\infty \quad \text { the line } l\left(b_{1}, b_{2}\right) \text { through } b_{1} \text { and } b_{2}, \\
0 \leqq \lambda<\infty & \text { the ray } r\left(b_{1}, b_{2}\right) \text { from } b_{1} \text { through } b_{2}, \\
0 \leqq \lambda \leqq 1 & \text { the segment } s\left(b_{1}, b_{2}\right) \text { from } b_{1} \text { to } b_{2} .
\end{array}
$$

A flat is the translate of a subspace and a hyperplane is an $(m-1)$-dimensional flat. The hyperplane $H$ is a hyperplane of support of $\bar{S}\left(b_{0}, \rho\right)$ if $\partial S\left(b_{0}, \rho\right) \cap H$ is not empty but $S\left(b_{0}, \rho\right) \cap H$ is empty. If $\bar{S}\left(b_{0}, \rho\right)$ possesses a unique hyperplane $H$ of support at a point $b \in \partial S\left(b_{0}, \rho\right)$, then $H$ is a tangent plane of $\bar{S}\left(b_{0}, \rho\right)$ at $b$.

Presented to the Society, September 1, 1966; received by the editors October 17, 1966.

( $\left.{ }^{1}\right)$ The preparation of this paper was supported by the National Science Foundation under grant GP-4052 and by the Air Force Office of Scientific Research under AF49(639)-1616. 
The space $B^{m}$ is a Minkowski space if the closed unit ball $\bar{S}(0,1)$ is strictly convex and $B^{m}$ is smooth if $\bar{S}(0,1)$ possesses a tangent plane at each point of $\partial S(0,1)$.

Let $\mathscr{F}$ be a closed subset of $B^{m}$. The distance of $b \in B^{m}$ from $\mathscr{F}$ is

$$
\|b-\mathscr{F}\|=\min _{f \in \mathscr{F}}\|f-b\| \text {. }
$$

The projection operator $\boldsymbol{P}_{\mathscr{F}}$ associates with each point $b \in B^{m}$ the set

$$
\boldsymbol{P}_{\mathscr{F}}(b)=\{f \mid f \in \mathscr{F},\|b-f\|=\|b-\mathscr{F}\|\} .
$$

The subscript $\mathscr{F}$ may be deleted when no ambiguity arises. The points $\boldsymbol{P}_{\mathscr{F}}(b)$ are projections of $b$ onto $\mathscr{F}$.

We begin the less standard definitions with

DefinItION 1. Let $l$ and $k$ be two lines in $B^{m}$ which intersect at $b_{0}$. The line $l$ is perpendicular to $k$ (written $l \perp k$ ) if

$$
b_{0}=\boldsymbol{P}_{l}(b)
$$

for every $b \in k$. If $l \perp k$ then $k$ is transversal to $l$.

If $L$ is a flat, then $l \perp L$ means that $l$ is perpendicular to every line in $L$. Perpendicularity is not symmetric in general and it is known, for $m>2$, that complete symmetry of perpendicularity implies that $B^{m}$ is a Hilbert space.

One may establish [1].

Lemma 1. Let $\mathscr{F} \subset B^{m}, b_{0} \notin \mathscr{F}$ and $p \in \boldsymbol{P}_{\mathscr{F}}\left(b_{0}\right)$. Then

(i) $p \in \boldsymbol{P}_{\mathscr{F}}(b)$ for every $b \in s\left(p, b_{0}\right)$,

(ii) if $B^{m}$ is Minkowskian, then

$$
p=\boldsymbol{P}_{\mathscr{F}}(b)
$$

for every $b=(1-\lambda) p+\lambda b_{0}$ with $0 \leqq \lambda<1$.

It is very useful to have a measure of "angle" between two lines. It appears difficult to define such a concept in Minkowski geometry which has the many properties of angle in Euclidean geometry. Let $l$ and $k$ intersect at $b_{0}$ and let

$$
\partial S\left(b_{0}, 1\right) \cap l=\left\{b_{1}, b_{2}\right\}, \quad \partial S\left(b_{0}, 1\right) \cap k=\left\{c_{1}, c_{2}\right\} .
$$

DEFINITION 2. The angle between two lines $l$ and $k$ which intersect is

$$
\theta(l, k)=\min \left\|b_{i}-c_{j}\right\|, \quad i, j=1,2 .
$$

For a general development of Minkowski geometry, one would replace the distance $\left\|b_{i}-c_{j}\right\|$ by the geodesic distance from $b_{i}$ to $c_{j}$ on the sphere $\partial S\left(b_{0}, 1\right)$. However, the present definition leads to considerable technical simplification in this context. The next lemma establishes some basic properties of angles and triangles. We consider the triangle formed by the points $b_{1}, b_{2}$, and $b_{3}$ and set

$$
l_{12}=l\left(b_{1}, b_{2}\right), \quad l_{23}=l\left(b_{2}, b_{3}\right), \quad l_{13}=l\left(b_{1}, b_{3}\right) .
$$


Lemma 2. Assume that $B^{m}$ is Minkowskian.

(i) The angle $\theta$ of Definition 2 is a metric.

(ii) Let $l_{23} \perp l_{12}$, then

$$
\left\|b_{2}-b_{3}\right\| \leqq\left\|b_{1}-b_{3}\right\| \theta\left(l_{12}, l_{13}\right) .
$$

(iii) Let $l_{23} \perp l_{12}$. Then there is a constant $0<K<\infty$ so that

$$
\left\|b_{2}-b_{3}\right\| \leqq K\left\|b_{1}-b_{2}\right\| \theta\left(l_{12}, l_{13}\right) \text {. }
$$

(iv) Let $\mathscr{C}$ be a closed cone in $B^{m}$ with vertex $b_{1}$, assume $b_{3} \notin \mathscr{C}$ and let $b_{2} \in \boldsymbol{P}_{\mathscr{C}}\left(b_{3}\right)$. Then there is an $\eta, 0<\eta \leqq 1$, so that

$$
\theta\left(l_{13}, l_{12}\right) \leqq \eta \theta\left(l_{13}, l\left(b_{1}, b\right)\right)
$$

for any $b \in \mathscr{C}$.

(v) Let $\left\|b_{1}-b_{2}\right\|=\left\|b_{1}-b_{3}\right\|=1$. Then there is a constant $\eta>0$ so that

$$
\left\|b_{3}-l_{12}\right\| \geqq \eta \theta\left(l_{12}, l_{13}\right) \text {. }
$$

Proof. (i) It is clear that $\theta(l, k)=0$ if and only if $l=k$. Consider three lines $l_{1}, l_{2}, l_{3}$ and associated points $b_{i}, c_{i}$, and $d_{i}, i=1,2$. Since $\partial S\left(b_{0}, 1\right)$ is centrally symmetric, we may assume that the points $c_{i}$ which minimize $\left\|b_{j}-c_{i}\right\|$ and $\left\|c_{i}-d_{j}\right\|(i, j=1,2)$ are the same, say $c_{1}$ for concreteness. Then

$$
\left\|b_{i}-d_{j}\right\| \leqq\left\|b_{i}-c_{1}\right\|+\left\|c_{1}-d_{j}\right\|
$$

for any $i$ and $j$ and the triangle inequality is established.

(ii) Let

$$
b_{2}^{\prime}=b_{1}+\left(b_{2}-b_{1}\right) /\left\|b_{3}-b_{1}\right\|, \quad b_{3}^{\prime}=b_{1}+\left(b_{3}-b_{1}\right) /\left\|b_{3}-b_{1}\right\| .
$$

Then $b_{3}^{\prime} \in \partial S\left(b_{1}, 1\right)$ and $b_{2}^{\prime}$ is a closest point on $l_{12}$ to $b_{3}^{\prime}$. Hence

$$
\theta\left(l_{13}, l_{12}\right) \geqq\left\|b_{3}^{\prime}-b_{2}^{\prime}\right\|=\left\|b_{3}-b_{2}\right\| /\left\|b_{3}-b_{1}\right\|
$$

which establishes the inequality.

(iii) Write the inequality as

$$
\left\|b_{2}-b_{3}\right\| /\left\|b_{1}-b_{2}\right\| \leqq K \theta\left(l_{12}, l_{13}\right)
$$

and then normalize the triangle so that $\left\|b_{1}-b_{2}\right\|=1$. The line $l_{13}$ intersects $\partial S\left(b_{1}, 1\right)$ at a point $b_{3}^{\prime}$ and

$$
\theta\left(l_{12}, l_{13}\right)=\left\|b_{2}-b_{3}^{\prime}\right\| \text {. }
$$

We now show that there is an $\eta$ so that

$$
\left\|b_{2}-b_{3}^{\prime}\right\| /\left\|b_{2}-b_{3}\right\| \geqq \eta>0 .
$$

Set $b_{2}^{\prime}=b_{1}+\left(b_{2}-b_{1}\right) /\left\|b_{1}-b_{3}^{\prime}\right\|$ and then $\left\|b_{2}-b_{3}\right\| \leqq\left\|b_{2}^{\prime}-b_{3}\right\|$ or

$$
\left\|b_{2}-b_{3}^{\prime}\right\| /\left\|b_{2}-b_{3}\right\| \geqq 1 /\left\|b_{1}-b_{3}\right\| .
$$


If $\left\|b_{1}-b_{3}\right\|<1$, then $\eta=1$ suffices. If $\left\|b_{1}-b_{3}\right\| \geqq 1$, then

$$
\frac{1}{\left\|b_{1}-b_{3}\right\|} \geqq \frac{1}{1+\left\|b_{2}-b_{3}^{\prime}\right\|+\left\|b_{2}-b_{3}\right\|} .
$$

For $b_{2} \neq b_{3}$, the number $\left\|b_{2}-b_{3}^{\prime}\right\| /\left\|b_{2}-b_{3}\right\|$ is a continuous positive function of $b_{2}$ and $b_{3}$. Further

$$
\operatorname{Lim}_{\left\|b_{2}-b_{3}\right\| \rightarrow 0} \frac{\left\|b_{2}-b_{3}^{\prime}\right\|}{\left\|b_{2}-b_{3}\right\|}=1
$$

and hence $\left\|b_{2}-b_{3}^{\prime}\right\| /\left\|b_{2}-b_{3}\right\|$ is a continuous positive function for all $b_{2}, b_{3}$ in the compact set $\partial S\left(b_{1}, 1\right)$. Thus it has a positive minimum value which we take for $\eta$. Set $K=1 / \eta$ and the desired inequality is established.

(iv) We assume that the triangle is normalized so that $\left\|b_{1}-b_{3}\right\|=1$. Then for $b \in \partial S\left(b_{1}, 1\right) \cap \mathscr{C}$ we have

Since

$$
\theta\left(l_{13}, l\left(b_{1}, b\right)\right)=\left\|b-b_{3}\right\| \geqq\left\|b_{2}-b_{3}\right\| .
$$

$$
\theta\left(l_{12}, l_{13}\right)=\frac{\left\|\left(b_{1}-b_{3}\right)\right\| b_{2}-b_{1}\left\|+b_{2}-b_{1}\right\|}{\left\|b_{2}-b_{1}\right\|}
$$

we may take

$$
\eta=\frac{\left\|b_{2}-b_{1}\right\|\left\|b_{2}-b_{3}\right\|}{\left\|\left(b_{1}-b_{3}\right)\right\| b_{2}-b_{1}\left\|+b_{2}-b_{1}\right\|}>0
$$

Simple examples show that one cannot always take $\eta=1$.

(v) Consider $b_{1}$ fixed and the function

$$
\phi\left(b_{2}, b_{3}\right)=\frac{\left\|b_{3}-l_{12}\right\|}{\theta\left(l_{12}, l_{13}\right)}=\frac{\left\|b_{3}-b_{2}^{\prime}\right\|}{\left\|b_{3}-b_{2}\right\|}
$$

where $b_{2}^{\prime}$ is the closest point in $l_{12}$ to $b_{3}$. Clearly $\phi \leqq 1$ and $\phi>0$ for $b_{2} \neq b_{3}$. Set

and note that

$$
b_{1}^{\prime}=b_{2}+\left(b_{3}-b_{2}\right) /\left\|b_{2}-b_{2}^{\prime}\right\|
$$

$$
\left\|b_{1}^{\prime}-b_{1}\right\|=\left\|b_{2}^{\prime}-b_{3}\right\| /\left\|b_{2}^{\prime}-b_{2}\right\| .
$$

If $\phi$ tends to zero with $\left\|b_{2}-b_{3}\right\|$ then, since

$$
\left\|b_{2}-b_{3}\right\|-\left\|b_{2}^{\prime}-b_{3}\right\| \leqq\left\|b_{2}^{\prime}-b_{2}\right\| \leqq\left\|b_{2}-b_{3}\right\|+\left\|b_{2}^{\prime}-b_{3}\right\|,
$$

we have that $\left\|b_{1}^{\prime}-b_{1}\right\|$ tends to zero. Now $b_{1}^{\prime} \in l_{23}$ and hence $b_{1}^{\prime}$ tends to the tangent plane of $\partial S\left(b_{1}, 1\right)$ at $b_{3}$. This implies that the center of this sphere is in a tangent plane of the sphere, which is contradictory. Hence $\phi\left(b_{2}, b_{3}\right)$ is bounded from zero as $\left\|b_{2}-b_{3}\right\|$ tends to zero and $\phi\left(b_{2}, b_{3}\right)$ is then a positive continuous function for $b_{2}, b_{3}$ in the compact set $\partial S\left(b_{1}, 1\right)$. We take $\eta$ to be the minimum value of $\phi$ and the proof is complete. 
DEFINITION 3. The line $l_{0}$ is a line of support of the set $\mathscr{F}$ at $f$ if there exist sequences $\left\{f_{i}\right\},\left\{g_{i}\right\} \subset \mathscr{F}$ so that

$$
l_{0}=\operatorname{Lim}_{f_{i} \rightarrow f: g_{i} \rightarrow f} l\left(f_{i}, g_{i}\right)
$$

The support cone of $\mathscr{F}$ at $f$ is the union of all lines of support of $\mathscr{F}$ at $f$.

Note that this double limiting process is stronger than usual definitions of this type and immediately implies that the support cone depends continuously on $f$ in the following sense: Denote the support cone at $f$ by $\mathscr{C}(f)$. Then given $f \in \mathscr{F}$ and $\varepsilon>0$ there is a $\delta>0$ so that $\|f-g\|<\delta$ implies that

$$
\max _{l \in \mathscr{C}(g)} \min _{k \in \mathscr{C}(f)} \theta(l, k)<\varepsilon .
$$

This definition is preliminary to defining tangent planes. Evidently, the support cone is a closed, connected set of dimension at least that of $\mathscr{F}$ at $f$, but such results are not required here. The following result is pertinent. We denote by $o(x)$ a function such that

$$
\operatorname{Lim}_{x \rightarrow 0}(o(x) /|x|)=0 .
$$

THEOREM 1. Let $\mathscr{C}$ be the support cone of $\mathscr{F}$ at $f$. Then for $g \in \mathscr{F}$

$$
\|g-\mathscr{C}\|=o(\|g-f\|)
$$

as $g$ tends to $f$.

Proof. Suppose that the result is false. Then there is a sequence $\left\{f_{i} \mid f_{i} \rightarrow f\right\}$ and an $\eta>0$ so that

$$
\left\|f_{i}-c_{i}\right\| \geqq \eta\left\|f_{i}-f\right\|
$$

where $c_{i} \in \boldsymbol{P}_{\mathscr{C}}\left(f_{i}\right)$. Consider the triangle formed by $f, c_{i}$, and $f_{i}$. We have that $l\left(f_{i}, c_{i}\right)=l_{1 i} \perp l\left(f, c_{i}\right)=l_{2 i}$. Hence, with $l_{3 i}=l\left(f_{i}, f\right)$ we have from Lemma 2 that

$$
\left\|f_{i}-c_{i}\right\| \leqq\left\|f-c_{i}\right\| \theta\left(l_{2 i}, l_{3 i}\right)
$$

or

$$
\eta\left\|f-f_{i}\right\| \leqq\left\|f_{i}-c_{i}\right\| \leqq \frac{\left\|f-f_{i}\right\| \theta\left(l_{2 i}, l_{3 i}\right)}{1-\theta\left(l_{2 i}, l_{3 i}\right)}
$$

This implies that

$$
0<\frac{\eta}{1+\eta} \theta\left(l_{2 i}, l_{3 i}\right)
$$

and the lines $l_{2 i}$ are bounded away from the lines $l_{3 i}$.

Since $l_{1 i} \perp l_{2 i}$ it follows from Lemma 2 (iv) that there is a $\mu>0$ so that for any $c \in \mathscr{C}$

$$
\theta\left(l_{2 i}, l_{3 i}\right) \leqq \mu \theta\left(l_{3 i}, l(f, c)\right)
$$


This implies that

$$
\theta\left(l_{3 i}, l(f, c)\right) \geqq \eta / \mu(1+\eta)>0
$$

for all $c \in \mathscr{C}$. Thus the limit of any convergent subsequence of $\left\{l_{3 i}\right\}$ is not contained in $\mathscr{C}$. This contradicts the definition of $\mathscr{C}$ and establishes the theorem.

Definition 4. Let $\mathscr{F} \subset B^{m}$ be $n$-dimensional at $f$. Then $\mathscr{F}$ is smooth at $f$ if the support cone of $\mathscr{F}$ at $f$ is an n-dimensional flat $T(f)$. The flat $T(f)$ is called the tangent plane of $\mathscr{F}$ at $f$.

If $\mathscr{F}$ is smooth at every point, then $\mathscr{F}$ is said to be smooth. Smoothness of the space $B^{m}$ is thus equivalent to smoothness of $\partial S(0,1)$. It appears likely that the word flat is superfluous in this definition. The remark following Definition 3 implies that the tangent plane varies continuously if $\mathscr{F}$ is smooth.

If $\mathscr{F}$ is a submanifold of class $C^{1}$ of $B^{m}$, then $\mathscr{F}$ has a tangent space at each point. It is apparent that the tangent plane defined above may be taken as a concrete realization of the tangent space. This fact induces naturally a norm into the tangent space and makes it a Banach space. Furthermore, the tangent space (as a Banach space) inherits many properties from the space $B^{m}$ (e.g., smoothness, strict convexity). The standard definition of the tangent space is somewhat different and thus some analysis is required to show that the tangent plane and tangent space of a submanifold $\mathscr{F}$ may be identified.

The primary use of the tangent plane is as a "local approximation." The next theorem establishes a fundamental property in this respect and is complementary to Theorem 1.

THEOREM 2. In the Minkowski space $B^{m}$ let $\mathscr{F}$ be smooth at $f$ and $l$ be a line through $f$ in the tangent plane $T(f)$ of $\mathscr{F}$ at $f$. Then, for $b \in l$, we have

$$
\|b-\mathscr{F}\|=o(\|b-f\|)
$$

as $b$ tends to $f$.

Proof. Suppose that the result is false. Then there is a sequence $\left\{b_{i} \mid b_{i} \in l, b_{i} \rightarrow f\right\}$ and an $\eta>0$ so that

$$
\left\|b_{i}-\mathscr{F}\right\| \geqq \eta\left\|b_{i}-f\right\| \text {. }
$$

Consider a point $g \in \partial S\left(f,\left\|b_{i}-f\right\|\right) \cap \mathscr{F}$. We have that

$$
\left\|b_{i}-g\right\| \geqq \eta\left\|b_{i}-f\right\|, \quad \theta(l, l(f, g))=\frac{\left\|b_{i}-g\right\|}{\left\|b_{i}-f\right\|} \geqq \eta>0 .
$$

Now since $\mathscr{F}$ is smooth, the tangent plane $T(f)$ is the union of all lines

$$
\operatorname{Lim}_{\left\|b_{g}-f\right\| \rightarrow 0} l(f, g)
$$

for all such $g$. This implies that $l \notin T(f)$, which is contradictory and establishes the result.

Examples show that this result is not always valid if $\mathscr{F}$ is not smooth at $f$. 
COROLlaRY. Let $\mathscr{F}$ be a smooth subset of the Minkowski space $B^{m}$ and let $G$ be a compact subset of $\mathscr{F}$. Then, with the notation of Theorems 1 and 2,

$$
\|b-\mathscr{F}\|=o(\|b-f\|), \quad\|g-T(f)\|=o(\|g-f\|)
$$

uniformly in $G$.

Proof. We consider the second relation only; the first follows in a similar manner. Let $\phi(f, \delta)$ denote the maximum angle $\theta(l(f, b), l(f, g))$ for $b \in T(f)$ and $g \in \mathscr{F}$ and $\|f-g\| \leqq \delta$. We extend the definition to $\delta=0$ by continuity, i.e., $\phi(f, 0)=0$. Since $\mathscr{F}$ is smooth, the tangent plane $T(f)$ depends continuously on $f$ and thus $\phi(f, \delta)$ is a continuous function of the variables $f$ and $\delta$ defined on the compact product space of $G$ and $[0,1]$. Hence $\phi(f, \delta)$ is uniformly continuous, and given $\varepsilon>0$ there is an $\eta>0$ so that $\delta<\eta$ implies that $|\phi(f, \delta)-\phi(f, 0)|=\phi(f, \delta)<\varepsilon$. Since $\|g-T(f)\| \leqq\|g-f\| \phi(f, \delta)$ the result is established.

The following technical result is required later. Let $S=S(b, 1)$ and let $H$ be a hyperplane of support of $\bar{S}$ at $f \in \partial S$. For any flat $T$ which contains $f$ set

$$
\theta(T)=\max _{t \in T} \min _{h \in H} \theta(l(f, t), l(f, h)) .
$$

This might be interpreted as the "angle" from $T$ to $H$.

Lemma 3. Let $B^{m}$ be a smooth Minkowski space. If $\theta(T)>0$ then there is a $t^{*} \in T$ with $\left\|f-t^{*}\right\|=1$ and $a \lambda_{0}>0$ (independent of $f$ ) so that for $0<\lambda<\lambda_{0}$,

$$
f+\lambda\left(t^{*}-f\right) \in S(b, 1) .
$$

Proof. Choose $t^{*} \in T$ and $h^{*} \in H$ at distance 1 from $f$ so that

$$
\theta(T)=\theta\left(l\left(f, t^{*}\right), l\left(f, h^{*}\right)\right) .
$$

The choice of $t^{*}$ is not unique and we choose $t^{*}$ so that $t^{*}$ is on the same side of $H$ as $b$. There is a $\lambda_{1}>0$ so that for $\lambda \leqq \lambda_{1}$ the line through $f+\lambda\left(t^{*}-f\right)$ perpendicular to $H$ intersects $\partial S$ in two points. Let $g(\lambda)$ denote the one closest to $H$ i.e., $l\left(f+\lambda\left(t^{*}-f\right), g(\lambda)\right) \perp H$.

From Lemma 2 to have an $\eta>0$ so that

$$
\left\|f+\lambda\left(t^{*}-f\right)-H\right\| \geqq \eta \lambda \theta(T)
$$

and from Theorem 1 we have

$$
\|g(\lambda)-H\|=o(\|g(\lambda)-f\|) .
$$

Given $\varepsilon>0$ there is a $\delta(\varepsilon)>0$ so that for $\|g(\lambda)-f\|<\delta(\varepsilon)$ we have

$$
o(\|g(\lambda)-f\|) \leqq \varepsilon\|g(\lambda)-f\| .
$$

Thus for $\|g(\lambda)-f\|<\delta(\varepsilon)$ we have

$$
\|g(\lambda)-H\| \leqq \varepsilon[\|h(\lambda)-f\|+\|g(\lambda)-H\|]
$$


where $h(\lambda)=l\left(f+\lambda\left(t^{*}-f\right), g(\lambda)\right) \cap H$. It is seen that

$$
\|h(\lambda)-f\| \leqq \lambda(1+\eta \theta(T))
$$

and if we take

$$
\varepsilon_{0}=\eta \theta(T) /(2+(\eta+2) \theta(T)),
$$

then for $\|g(\lambda)-f\| \leqq \delta\left(\varepsilon_{0}\right)$ we have

$$
\|g(\lambda)-H\| \leqq \eta \lambda \theta(T) / 2 .
$$

It is clear that $\|g(\lambda)-f\|$ tends to zero as $\lambda$ tends to zero. Thus given $\delta>0$ there is a $\lambda(\delta)>0$ so that $\lambda \leqq \lambda(\delta)$ implies that $\|g(\lambda)-f\| \leqq \delta$. Take $\lambda_{0}=\lambda\left(\delta\left(\varepsilon_{0}\right)\right)$ and then for $0<\lambda<\lambda_{0}$

$$
\|g(\lambda)-H\| \leqq \lambda \eta \theta(T) / 2<\left\|f+\lambda\left(t^{*}-f\right)-H\right\|
$$

and hence $f-\lambda\left(t^{*}-f\right) \in S$. This concludes the proof.

Let $T_{i}$ and $U$ be subspaces of $B^{m}$.

LEMMA 4. Assume that

$$
\operatorname{Lim}_{i \rightarrow \infty} T_{i}=U
$$

and $\left\{l_{i}\right\}$ is a sequence of lines with $l_{i} \perp T_{i}$. If $l$ is any limiting line of $\left\{l_{i}\right\}$ then $l \perp U$.

Proof. We may assume that the $l_{i}$ pass through the origin 0 of $B^{m}$ since perpendicularity is unaffected by translation. Given $l$, take $\left\{l_{i}\right\}$ to be a convergent subsequence with limit $l$ and choose points $b \in l, b_{i} \in l_{i}$ with $\|b\|=\left\|b_{i}\right\|=1$ and $b_{i} \rightarrow b$. Suppose that for

$$
u \in \boldsymbol{P}_{U}(b)
$$

we have $\|u-b\|<1$. Denote by $t_{i}$ points in $T_{i}$ closest to $u$ with $\left\|t_{i}\right\|=\|u\|$. Then

$$
\left\|b_{i}-t_{i}\right\| \leqq\left\|b_{i}-b\right\|+\|b-u\|+\left\|u-t_{i}\right\| .
$$

Now $\left\|t_{i}-u\right\|$ tends to zero since $T_{i}$ tends to $U$ and

$$
\left\|b_{i}-t_{i}\right\| \leqq\|b-u\|+o(1) .
$$

For $i$ sufficiently large this contradicts $l_{i} \perp T_{i}$ and establishes the lemma.

The next theorem establishes the intuitively desirable result that projections on a smooth submanifold are also projections onto the tangent planes of the submanifold.

THEOREM 3. Assume that $\mathscr{F}$ is smooth at $f$ and

$$
f \in \boldsymbol{P}_{\mathscr{F}}(b) .
$$

Denote by $T(f)$ the tangent plane of $\mathscr{F}$ at $f$. Then $l(b, f) \perp T(f)$.

Proof. Assume the contrary, i.e., that $f$ is not a projection of $b$ onto $T(f)$. Let $t$ be such a projection and consider a point $c$ in the interior of $s(f, b)$ and denote by $t_{1}$ its projection onto $T$. See Figure 1. Further choose

$$
g \in \boldsymbol{P}_{\mathscr{F}}\left(t_{1}\right) \text {. }
$$


If $B^{m}$ is not strictly convex then $t$ and $t_{1}$ might not be uniquely determined. However once $t$ is chosen, the point $t_{1}$ may be chosen so that

$$
\left\|t_{1}-f\right\| /\|c-f\|=\|t-f\| /\|b-f\| \text {. }
$$

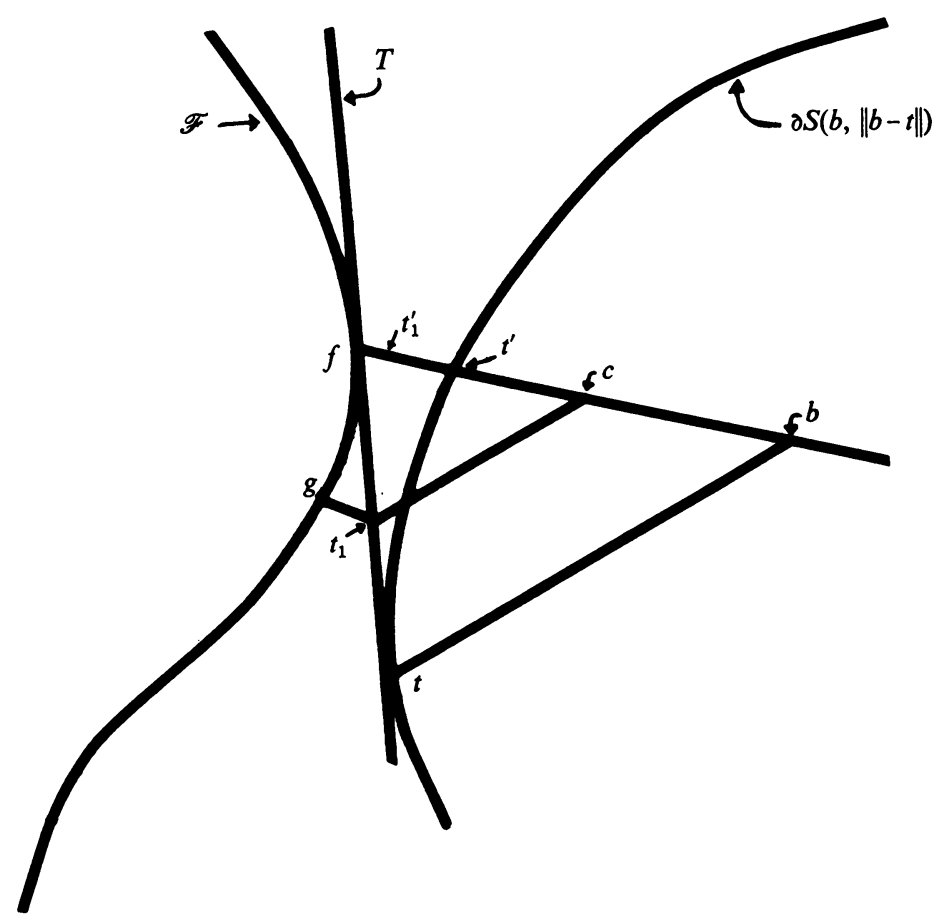

Figure 1. The construction of Theorem 3

The points $t^{\prime}, t_{1}^{\prime}$ are determined on the segment $s(b, f)$ so that

$$
\|b-t\|=\left\|b-t^{\prime}\right\|, \quad\left\|c-t_{1}\right\|=\left\|c-t_{1}^{\prime}\right\| .
$$

We have from Theorem 2 and the triangle inequality that, as $\left\|f-b_{1}\right\| \rightarrow 0$,

$$
\frac{\|c-g\|}{\left\|c-t_{1}\right\|} \leqq 1+\frac{o\left(\left\|f-t_{1}\right\|\right)}{\left\|c-t_{1}\right\|}
$$

Since $\left\|f-t^{\prime}\right\|>0$, we have that $\left\|f-t_{1}\right\|\left\|f-t^{\prime}\right\|=\|f-t\|\left\|f-t_{1}^{\prime}\right\|$ and

$$
o\left(\left\|f-t_{1}\right\|\right)=o\left(\left\|f-t_{1}^{\prime}\right\|\right) .
$$

We have from Lemma 1 and our contrary assumption that

$$
\frac{\|c-g\|}{\left\|c-t_{1}\right\|} \geqq \frac{\|c-f\|}{\left\|c-t_{1}\right\|}=1+\frac{\left\|f-t_{1}^{\prime}\right\|}{\left\|c-t_{1}\right\|} .
$$

These three relations combine to give

$$
1+\frac{o\left(\left\|f-t_{1}^{\prime}\right\|\right)}{\left\|c-t_{1}\right\|} \geqq 1+\frac{\left\|f-t_{1}^{\prime}\right\|}{\left\|c-t_{1}\right\|}
$$


as $\left\|f-t_{1}^{\prime}\right\|$ tends to zero (i.e., as $c$ tends to $f$ ). This is impossible and thus the contrary assumption is untenable.

The following useful result is readily established [1].

Lemma 5. Let $T$ be a flat in the Minkowski space $B^{m}$. Then every point $b \in B^{m}$ has a unique representation

$$
b=t+t^{\perp},
$$

where $t=\boldsymbol{P}_{T}(b) \in T$ and $t^{\perp}=(b-t)$ is perpendicular to $T$.

We define normals and normal rays of a submanifold of $B^{m}$ as follows.

DEFINITION 5. Let $T(f)$ be the tangent plane of $\mathscr{F}$ at $f$. If $f \in l$ and $l \perp T(f)$, then $l$ is a normal line of $\mathscr{F}$ at $f$. The set of normal lines of $\mathscr{F}$ at $f$ is the normal set of $\mathscr{F}$ at $f$. If $l(f, b) \perp T(f)$, then the ray $r(f, b)$ is the normal ray of $\mathscr{F}$ at $f$ through $b$.

Note that if $\mathscr{F}$ is smooth then the tangent plane and normal set of $\mathscr{F}$ at $f$ depend continuously on $f$. Simple examples in $B^{3}$ show that the normal set need not be a flat. Other simple properties of normals are given by the following readily established lemma.

LEMMA 6. Let $T$ be a subspace of the Minkowski space $B^{m}$ and let $N(t)$ be the normal set of $T$ at $t$. Then

$$
N(t)=N(0)+t .
$$

If $T$ is a line then $N(0)$ separates $B^{m}$ into two arcwise connected sets.

3. Curvature in Minkowski geometry. We are interested in determining conditions under which a point $b \in B^{m}$ has a unique projection on a submanifold. Simple examples in $B^{2}$ show that smoothness of $\mathscr{F}$ is not sufficient for such a study nor is the knuwledge of the Euclidean curvature of $\mathscr{F}$ at a point $f$. Attempts to define an adequate concept of curvature in non-Riemannian spaces have not been particularly successful. As pointed out by Buseman [1], the reason for this is that curvature plays many roles in Riemannian geometry and it is unreasonable to expect that there is a single concept which plays all these roles in a more general context.

Intuitively, we are interested in measuring how "fast" $\mathscr{F}$ is bending with respect to the spheres in $B^{m}$. For one-dimensional submanifolds of a Minkowski space $B^{2}$, the classical intrinsic definition of curvature can be directly generalized. In $B^{2}$ denote by $C\left(b_{1}, b_{2}, b_{3}\right)$ the circle (the boundary of a ball) which passes through $b_{1}, b_{2}$, and $b_{3}$ and denote its radius by $\rho\left(b_{1}, b_{2}, b_{3}\right)$. We have

Definition 6. Let $B^{2}$ be a Minkowski 2-space. The curve $\mathscr{F} \subset B^{2}$ is said to have radius of curvature $\rho$ at $f \in \mathscr{F}$ if

$$
\rho=\operatorname{Lim}_{b_{1}, b_{2}, b_{3} \rightarrow f} \rho\left(b_{1}, b_{2}, b_{3}\right) .
$$

The curvature of $\mathscr{F}$ at $f$ is $1 / \rho$. 
Note that this definition involves the intrinsic nature of $B^{2}$ much more deeply than does that of the tangent line.

This definition does not readily generalize for $n>2$ and we use rather the following construction. Let $r(f, b)$ be a normal ray of $\mathscr{F}$ at $f$ through $b$. For any sequence $\left\{f_{i} \mid f_{i} \in \mathscr{F}\right\}$ we associate a sequence $\partial S_{i}=\partial S_{i}\left(b_{i}, \rho_{i}\right)$ where $b_{i} \in r, \rho_{i}=\left\|f-b_{i}\right\|$ and $f_{i} \in \partial S_{i}$. If such a sphere $\partial S_{i}$ does not exist, we set $\rho_{i}=+\infty$.

DEFINITION 7. The radius of curvature of $\mathscr{F}$ at $f$ in the direction $r(f, b)$ is $\rho(f, r)$ if (i) for every sequence $\left\{f_{i}\right\}$ so that $f_{i} \rightarrow f$ we have

$$
\operatorname{Liminf}_{f_{i} \rightarrow f} \rho_{i} \geqq \rho(f, r)
$$

and (ii) for some sequence $\left\{f_{i}\right\}$ so that $f_{i} \rightarrow f$ we have

$$
\operatorname{Lim}_{f_{i} \rightarrow f} \rho_{i}=\rho(f, r) .
$$

The curvature $\sigma(f, r)$ of $\mathscr{F}$ at $f$ in the normal direction $r(f, b)$ is $1 / \rho(f, r)$. The curvature of $\mathscr{F}$ at $f$ is

$$
\sigma(f)=\max _{r} \rho(f, r)
$$

for all rays $r$ in the normal set of $\mathscr{F}$ at $f$.

In Euclidean geometry there is a well-known relationship between curvature and the contact of a submanifold with its tangent plane. Our results in Theorems 1 and 2 for smooth manifolds are the direct analogs of similar results in Euclidean space. However, simple examples show that the usual Euclidean relationship between curvature and order of contact does not extend to the present context in a familiar form.

In order to study this more carefully, we consider the unit sphere $\partial S(0,1)$ along with one of its tangent planes $H$ at $f$. Let $l$ be a line in $H$ through $f$ and for $h \in l$ take $g(h) \in \partial S$ so that

$$
h=\boldsymbol{P}_{H}(g(h)) .
$$

We assume that $B^{m}$ is Minkowskian and smooth, so that $H$ and $h$ are uniquely determined. Set $x=\|h-f\|$ and the function

$$
h_{1}(x)=\|g(h)-H\|
$$

measures the height of $\partial S$ above $H$. Consider another sphere $\partial S_{\rho}$ of radius $\rho$ and with $H$ as tangent plane at $f \in \partial S_{\rho}$. Denote the analogous height function by $h_{\rho}(x)$ and we see

$$
h_{\rho}(x)=\rho h_{1}(x / \rho) .
$$

The function $h_{1}(x)$ describes the contact of $\partial S$ with its tangent plane at $f$.

Let $\mathscr{F}$ have curvature $\sigma(f, r)<\infty$ at $f$ in the direction $r$. Then for some sequence $\left\{f_{i}\right\}$

$$
\operatorname{Lim}_{f_{i} \rightarrow f} 1 / \rho_{i}=\sigma(f, r)
$$


in the notation of Definition 7. Equivalently, as $i \rightarrow \infty$,

$$
\rho_{i}=\rho(f, r)(1+o(1)) \text {. }
$$

For this sequence consider points $g_{i} \in \partial S(b, \rho(f, r)) \cap l_{i}$ where $b \in r(f, b)$ and $l_{i}$ is a line parallel to $r$ passing through $f_{i}$.

THEOREM 4. Let $B^{m}$ be Minkowskian and smooth and let $\left\{f_{i}\right\}$ be a sequence in $\mathscr{F} \subset B^{m}$ so that (1) holds. If

$$
\operatorname{Lim}_{t \rightarrow 0} \frac{h_{1}(x(1+o(1)))}{h_{1}(x)}=1
$$

for any choice of $l$, then

$$
\left\|f_{i}-H\right\|=\left\|g_{i}-H\right\|+o\left(\left\|g_{i}-H\right\|\right) .
$$

Proof. From (1) we have with $\rho^{*}=\rho(f, r)$,

$$
h_{\rho_{i}}\left(x_{i}\right)=\frac{\rho^{*}}{1+o(1)} h_{1}\left(\frac{x_{i}}{\rho^{*}}(1+o(1))\right)=\left\|f_{i}-H\right\|
$$

where $x_{i}=\left\|f-\boldsymbol{P}_{H}\left(g_{i}\right)\right\|, g_{i} \in \partial S\left(b_{i}, \rho_{i}\right)$. Thus

$$
\begin{aligned}
\left\|f_{i}-H\right\| & =\rho^{*} h_{1}\left(\frac{x_{i}}{\rho^{*}}(1+o(1))\right)-\rho^{*} o(1) h_{1}\left(\frac{x_{i}}{\rho^{*}}(1+o(1))\right) \\
& =h_{\rho^{*}}\left(t_{i}\right) \frac{h_{1}\left(x_{i}(1+o(1)) / \rho^{*}\right)}{h_{1}\left(x_{i} / \rho^{*}\right)}+h_{\rho^{*}}\left(x_{i}\right)\left[o(1) \frac{h_{1}\left(x_{i}(1+o(1)) / \rho^{*}\right)}{h_{1}\left(x_{i} / \rho^{*}\right)}\right] .
\end{aligned}
$$

The hypothesis (2) on the behavior of $h_{1}(x)$ implies then

$$
h_{\rho_{i}}\left(x_{i}\right)=h_{\rho^{*}}\left(x_{i}\right)[1+o(1)]
$$

or, equivalently,

$$
\left\|f_{i}-H\right\|=\left\|g_{i}-H\right\|+o\left(\left\|g_{i}-H\right\|\right) .
$$

COROLlaRY 1. If (2) holds and $f_{i} \rightarrow f$, then

$$
\left\|f_{i}-H\right\| \leqq\left\|g_{i}-H\right\|+o\left(\left\|g_{i}-H\right\|\right)
$$

with the terminology of Theorem 4.

A short calculation establishes

COROLlaRY 2. If $h_{1}(x)$ is continuously differentiable and there is a constant $M<\infty$ so that

$$
\frac{1}{h_{1}(x)} \frac{d h_{1}(x)}{d t} \leqq \frac{M}{x}
$$

holds for all $x>0$ and all lines $l$, then

$$
\left\|f_{i}-H\right\| \leqq\left\|g_{i}-H\right\|+o\left(\left\|g_{i}-H\right\|\right)
$$

and equality holds for any sequence $\left\{f_{i}\right\}$ for which (1) is valid. 
COROllary 3. Let $\mathscr{F}$ be a submanifold of m-dimensional $L_{p}$ space, $1<p<\infty$, which has curvature $\sigma(f, r)<\infty$ at $f$ in the direction $r$. With the terminology of Theorem 4 we have

$$
\left\|f_{i}-H\right\| \leqq\left\|g_{i}-H\right\|+o\left(\left\|g_{i}-H\right\|\right)
$$

and equality holds for any sequence $\left\{f_{i}\right\}$ for which (1) is valid.

The following example shows that there are situations where $\mathscr{F}$ has curvature $\sigma(f, r)<\infty$ at $f$ and yet the order of contact of $\mathscr{F}$ with its tangent plane is different from the order of contact that the sphere $\partial S(b, 1 / \sigma), b \in r,\|b-f\|=1 / \sigma$, makes with its tangent plane. Take $B^{2}$ where the circle $\partial S((0,1), 1)$ is explicitly given by

$$
h_{1}(x)=e^{-1 /|x|^{p}}
$$

for $x \leqq x_{1}$. The curve $\mathscr{F}$ is given by

$$
f(x)=g(x) \exp \left(-[g(x) /|x|]^{p}\right) .
$$

For $g(x)=1+|x|^{q}, q<p$, we have

$$
\operatorname{Lim}_{x \rightarrow 0} \frac{\|f(x)-H\|}{\|g(x)-H\|}=0
$$

for $g(x)=1-|x|^{q}, q<p$ we have

$$
\operatorname{Lim}_{x \rightarrow 0} \frac{\|f(x)-H\|}{\|g(x)-H\|}=\infty .
$$

4. Projections on submanifolds. The principal questions concerning projections on submanifolds are those of the existence, uniqueness and characterization of projections. In this section we present several results pertinent to these questions. All but one, Theorem 7 , of these results are known or readily established.

THEOREM 5. Every point in $B^{m}$ has a projection on $\mathscr{F}$ if and only if $\mathscr{F}$ is closed.

DEFINITION 8. A sphere $\partial S(b, \rho)$ is a sphere of support of $\mathscr{F}$ at $f$ if $\mathscr{F} \cap S(b, \rho)$ is empty and $f \in \partial S(b, \rho)$.

THEOREM 6. We have

$$
f \in \boldsymbol{P}_{\mathscr{F}}(b)
$$

if and only if the sphere $\partial S(b,\|b-f\|)$ is a sphere of support of $\mathscr{F}$ at $f$.

The following are examples of direct corollaries of this theorem.

Corollary 1. Let $\mathscr{F}$ be convex. Then

$$
f \in \boldsymbol{P}_{\mathscr{F}}(b)
$$

if and only if $\mathscr{F}$ and $\partial S(b,\|b-\mathscr{F}\|)$ have a common hyperplane of support at $f$.

Corollary 2. Let $\mathscr{F}$ be smooth. Then

$$
f \in \boldsymbol{P}_{\mathscr{F}}(b)
$$


only if the tangent plane of $\mathscr{F}$ at $f$ is in a hyperplane of support of $\partial S(b,\|b-f\|)$ at $f$. Furthermore $b$ is in the normal set of $\mathscr{F}$ at $f$.

COROLlaRY 3. Let $\mathscr{F}$ be smooth and $B^{m}$ be a smooth Minkowski space. If

$$
f_{0} \in \boldsymbol{P}_{\mathscr{F}}(b),
$$

then $f_{0}$ is a critical point of $\|b-f\|[5]$.

THEOREM 7. Let $B^{m}$ be a Minkowski space and let $\mathscr{F} \subset B^{m}$ have bounded curvature. The sphere $\partial S(b,\|b-f\|)$ is a sphere of support of $\mathscr{F}$ at $f$ only if $r(f, b)$ is a normal ray and $\|b-f\| \leqq \rho(f, r)$. If $r(f, b)$ is a normal ray and $\|b-f\|<\rho(f, r)$, then there is an $\varepsilon>0$ such that $\partial S(b,\|b-f\|)$ is a sphere of support of $\mathscr{F} \cap S(f, \varepsilon)$ at $f$.

Proof. It is clear that $r(f, b)$ is a normal ray if $\partial S(b,\|b-f\|)$ is a sphere of support at $f$. Every sphere $\partial S\left(b^{\prime},\left\|b^{\prime}-f\right\|\right)$ with $b^{\prime} \in r(f, b)$ and $\left\|b^{\prime}-f\right\|<\|b-f\|$ is in $S(b,\|b-f\|)$ except for the point $f$. From Definition 7 we have a sequence $\left\{f_{i}\right\} \subset(F)$ and associated $b_{i} \in r(f, b), \rho_{i}=\left\|b_{i}-f\right\|$ so that $f_{i} \in \partial S\left(b_{i}, \rho_{i}\right)$ and

$$
\operatorname{Lim}_{f_{i} \rightarrow f} \rho_{i}=\rho(f, r) .
$$

Hence if $\|b-f\|>\rho(f, r)$ then for $i$ sufficiently large $S(b,\|b-f\|)$ contains every point $f_{i}$ and $\partial S(b,\|b-f\|)$ is not a sphere of support of $\mathscr{F}$ at $f$. This is contradictory and establishes the first statement of the theorem.

Assume that $\|b-f\|<\rho(f, r)$ and $r(f, b)$ is a normal ray of $\mathscr{F}$ at $f$. Suppose $\partial S(b,\|b-f\|)$ is not a sphere of support of $\mathscr{F} \cap S(f, 1 / i)$ for infinitely many $i$. Then for each of these $i, S(b,\|b-f\|)$ contains a point $f_{i} \in \mathscr{F}$ with $\left\|f_{i}-f\right\|<1 / i$. Consider the spheres $\partial S\left(b_{i}, \rho_{i}\right), b_{i} \in r(f, b), \rho_{i}=\left\|b_{i}-f\right\|$ which contain $f_{i}$. Clearly $\rho_{i}<\|b-f\|$. The sequence $\left\{f_{i}\right\}$ satisfies the requirements of Definition 7 and hence

$$
\rho(f, r) \leqq \operatorname{Liminf}_{f_{i} \rightarrow f} \rho_{i} \leqq\|b-f\| .
$$

This contradicts $\|b-f\|<\rho(f, r)$ and establishes the second statement.

A closed subset $\mathscr{F}$ of $B^{m}$ is called a Motzkin set (or a Chebyshev set) if every point $b \in B^{m}$ has a unique projection onto $\mathscr{F}$.

THEOREM 8. Let $\mathscr{F}$ be a closed submanifold of a smooth space $B^{m}$. Then $\mathscr{F}$ is a Motzkin set only if $\mathscr{F}$ is a linear manifold [8].

CoROllaRy 1. Let $\mathscr{F}$ be a closed submanifold of a smooth Minkowski space $B^{m}$. Then $\mathscr{F}$ is a Motzkin set if and only if $\mathscr{F}$ is a linear manifold.

Corollary 2. Let $F(A, x)$ be a nonlinear unisolvent approximating function, let $X$ be a real finite set and \|\|$_{p}$ denote an $L_{p}$ norm. Then, for $1<p<\infty$, there exists a function $b(x)$ defined on $X$ such that

$$
\left\|b(x)-F\left(A_{1}, x\right)\right\|_{p}=\left\|b(x)-F\left(A_{2}, x\right)\right\|_{p}=\inf _{A}\|b(x)-F(A, x)\|_{p}
$$

and $A_{1} \neq A_{2}$. 
COROLLARY 3. Let $\mathscr{F}$ be a nonlinear, closed, locally unisolvent approximating function, let $X$ be a real finite set and \|\|$_{p}$ and $L_{p}$ norm. Then, for $1<p<\infty$, there exists a function $b(x)$ defined on $X$ so that (3) holds and $A_{1} \neq A_{2}$.

See [6] and [7] for the terminology used in Corollaries 2 and 3.

5. The local uniqueness theorem. The principal result of this paper is to give sufficient conditions on a submanifold $\mathscr{F}$ in order that it have a neighborhood $\mathscr{F}_{u}$ of unique projection (i.e., $\boldsymbol{P}_{\mathscr{F}}(b)$ is a single point for $b \in \mathscr{F}_{u}$ ). It is clear from Theorem 5 that $\mathscr{F}$ must be closed (i.e., a closed set). It is believed that these conditions are also necessary.

Note that we use the differential topology definition of a submanifold (i.e., the topology on the submanifold is induced by the topology of the containing space $\left.B^{m}\right)$. This implies in particular that every neighborhood $N$ of $f$ in $\mathscr{F}$ contains $\mathscr{F} \cap S(f, \varepsilon)$ for some $\varepsilon>0$.

LEMMA 7. Let $\mathscr{F}$ be a closed smooth submanifold of $B^{m}$ and $f \in \mathscr{F}$. Then there is an $\varepsilon(f)>0$ such that $g \in \mathscr{F},\|f-g\| \leqq \varepsilon(f)$ implies that $S(g, \lambda) \cap \mathscr{F}$ is connected for all $\lambda \leqq \varepsilon(f)$.

Proof. Suppose to the contrary that there is a sequence $\left\{g_{i} \mid g_{i} \rightarrow f\right\}$ such that $S\left(g_{i}, \lambda_{i}\right) \cap \mathscr{F}$ is disconnected for some $\lambda_{i} \leqq\left\|g_{i}-f\right\|$. Let $E_{i}$ be a connected component of $S\left(g_{i}, \lambda_{i}\right) \cap \mathscr{F}$ which does not contain $g_{i}$. Since $\mathscr{F}$ is a closed manifold we have $\partial E_{i} \subset \partial S\left(g_{i}, \lambda_{i}\right)$ and hence there is a point $e_{i} \in E_{i}$ so that $\left\|g_{i}-E_{i}\right\|=\left\|g_{i}-e_{i}\right\|$. By Corollary 2 of Theorem 6 we have that $l_{i}=l\left(g_{i}, e_{i}\right)$ is perpendicular to the tangent plane $T\left(e_{i}\right)$ of $\mathscr{F}$ at $e_{i}$. Since $\mathscr{F}$ is smooth we have $T\left(e_{i}\right) \rightarrow T(f)$ and we have from Lemma 4 that any limit $l$ of $\left\{l_{i}\right\}$ has $l \perp T(f)$. Clearly such a limit exists and since $l_{i}$ interpolates $\mathscr{F}$ at $g_{i}$ and $e_{i}, l \subset T(f)$ by definition. This is a contradiction and establishes the result.

Corollary. Let $G$ be a compact subset of a closed, smooth submanifold $\mathscr{F}$. Then there is an $\varepsilon>0$, independent of $f$, so that for $f \in G, \lambda \leqq \varepsilon, S(f, \lambda) \cap \mathscr{F}$ is connected.

Proof. Let $\varepsilon(f)$ be as in Lemma 7. The set

$$
\bigcup_{f \in G} S(f, \varepsilon(f))
$$

covers $G$ and hence there is a finite set $f_{i}, i=1,2, \ldots, q$ so that $S\left(f_{i}, \varepsilon\left(f_{i}\right)\right)$ covers $G$. Take $\varepsilon=\min \left[\varepsilon\left(f_{i}\right)\right], i=1,2, \ldots, q$.

We consider a closed submanifold $\mathscr{F}$ of $B^{m}$ and compact connected subsets of $\mathscr{F}$ are denoted generically by $\Gamma$.

Lemma 8. Suppose $G$ is compact and $G \subset \Gamma_{1}$. Then for any $b \in B^{m}$ there exists a $\Gamma_{0}$ so that

$$
\left\|b-\Gamma_{0}\right\|=\inf \|b-\Gamma\|<\infty,
$$

where the infimum is over all $\Gamma$ which contain $G$. 
Proof. Denote inf $\|b-\Gamma\|$ by $d$ and let $\Gamma_{i}$ be the connected component of $\mathscr{F} \cap \bar{S}(b, d+1 / i)$ which contains $G$. It is clear that $d \leqq\left\|b-\Gamma_{1}\right\|<\infty$. Further $\Gamma_{i+1} \subset \Gamma_{i}$ and hence $\left\{\Gamma_{i}\right\}$ is a nested sequence of compact connected sets. Thus

$$
\Gamma_{0}=\bigcap_{i=2}^{\infty} \Gamma_{i}
$$

is a compact connected set and it clearly contains $G$. Since

$$
\left\|b-\Gamma_{0}\right\|=\operatorname{Lim}_{i \rightarrow \infty}\left\|b-\Gamma_{i}\right\|=d,
$$

the proof is complete.

We denote by $\Gamma^{*}$ the subset of $\Gamma$

$$
\Gamma^{*}=\{f \mid f \in \Gamma,\|b-f\|=\|b-\Gamma\|\} .
$$

LemMA 9. Let $B^{m}$ be a smooth Minkowski space and $\mathscr{F}$ be a smooth submanifold and $G$ be a compact subset of $\mathscr{F}$. If

$$
\|b-G\|<\left\|b-\Gamma_{0}\right\|=\inf \|b-\Gamma\|=d<\infty,
$$

where the infimum is taken over all $\Gamma$ containing $G$, then there is a point $f^{*} \in \Gamma_{0}^{*}$ so that $l\left(b, f^{*}\right) \perp T\left(f^{*}\right)$, where $T\left(f^{*}\right)$ is the tangent plane of $\mathscr{F}$ at $f^{*}$.

Proof. The proof is by contradiction. Let $H(f)$ denote the tangent plane of $\partial S(b,\|b-f\|)$ at $f$. Consider a point $f+t(f) \in T(f)$ such that $\|t(f)\|=1$ and $\|f+t(f)-H(f)\|$ is maximized. We choose $t(f)$ so that $f+t(f)$ is on the same side of $H(f)$ as $b$. By Lemma 5 each $t(f)$ may be written as $h(f)+n(f)$ where $f+h(f) \in H(f)$ and $n(f) \perp H(f)$. If $\|n(f)\|=0$ then $l(b, f) \perp T(f)$. Assume that $\|n(f)\|>0$ for each $f \in \Gamma_{0}^{*}$. Since $\|n(f)\|$ is continuous and $\Gamma_{0}^{*}$ is compact there is an $\eta$ so that $\|n(f)\| \geqq \eta>0$ for $f \in \Gamma_{0}^{*}$.

It follows from Lemma 3 that there is a $\lambda(\|b-f\|)$ so that for $0<\lambda<\lambda(\|b-f\|)$ we have

$$
f+\lambda t(f) \in S(b,\|b-f\|) .
$$

It is clear from the homothetic nature of the geometry that $\lambda(\|b-f\|)$ is proportional to $\|b-f\|$. Since $G$ and $\Gamma_{0}^{*}$ are compact and $\|b-G\|<\left\|b-\Gamma_{0}^{*}\right\|$ there is an $\varepsilon_{1}>0$ so that $\left\|f-\Gamma_{0}^{*}\right\| \leqq \varepsilon \leqq \varepsilon_{1}$ implies that $\|f-G\|>0$. We take $\varepsilon_{1}<1$ and set

$$
E=\left\{f \mid\left\|f-\Gamma_{0}^{*}\right\|<\varepsilon_{1}\right\}
$$

Take $\lambda_{0}=\min [\lambda(\|b-f\|)]$ for $\|b-E\| \leqq\|b-f\| \leqq d$. Further take

$$
\lambda_{1}=d-\max \|b-f\|>0
$$

with the maximum taken for $f \in \Gamma_{0}, f \notin E$.

For $f \in E$ we have the following consequences of Theorems 1 and 2. First

$$
\|c-H(f)\| \leqq o_{1}(\varepsilon), \quad c \in \bar{S}(f, \varepsilon) \cap \partial S(b,\|b-f\|) .
$$


Since the closure of $E$ is compact it follows that $o_{1}(\varepsilon)$ is independent of $f$. Likewise

$$
\|u-\mathscr{F}\| \leqq o_{2}(\varepsilon), \quad u \in \bar{S}(f, \varepsilon) \cap T(f)
$$

and $O_{2}(\varepsilon)$ is independent of $f$ for $f \in E$. The Corollary of Theorem 7 implies that there is an $\varepsilon_{0}>0$ so that for each $f \in \mathscr{F}$ if $\lambda \leqq \varepsilon_{0}$ then $S(f, \lambda) \cap \mathscr{F}$ is connected. Choose $\lambda_{2}$ so that $0<\lambda<\lambda_{2}$ implies that

Take

$$
o_{1}(\lambda), o_{2}(\lambda) \leqq \min \left[\varepsilon_{0}, \eta \lambda / 8\right]
$$

$$
\lambda^{*}=\frac{1}{2} \min \left[\lambda_{0}, \lambda_{1}, \lambda_{2}\right]
$$

and for $f \in \Gamma_{0}, f \notin E$ set

$$
U(f)=S\left(f, \eta \lambda^{*} / 2\right) \cap \mathscr{F} .
$$

We note for $f \in E$ that if $f_{1}, f_{2}$ are projections of $f+\lambda^{*} t(f)$ onto $\mathscr{F}$, then $\left\|f_{1}-f_{2}\right\|$ $\leqq \eta \lambda^{*} / 4$ since

$$
\left\|f+\lambda^{*} t(f)-\mathscr{F}\right\| \leqq \lambda \eta^{*} / 8 .
$$

Thus if we choose one such $f_{1}$ and for $f \in E$ set

then we have

$$
U(f)=S\left(f_{1}, \eta \lambda^{*} / 2\right) \cap \mathscr{F},
$$

$$
U(f) \supset \boldsymbol{P}_{\mathscr{F}}\left(f+\lambda^{*} t(f)\right)
$$

and $U(f)$ is a connected open set.

Consider $f \in E$ and choose

$$
f_{1} \in \boldsymbol{P}_{\mathscr{F}}\left(f+\lambda^{*} t(f)\right), \quad g_{1} \in \boldsymbol{P}_{\mathscr{F}}\left(g+\lambda^{*} t(g)\right) .
$$

We have

$$
\left\|f_{1}-g_{1}\right\| \leqq\left\|f_{1}-f-\lambda^{*} t(f)\right\|+\left\|g_{1}-g-\lambda^{*} t(f)\right\|+\left\|f+\lambda^{*} t(f)-g-\lambda^{*} t(g)\right\| .
$$

Since $t(f)$ is a continuous function on the closure of $E$, there is a $\delta_{1}>0$ so that $\|f-g\|<\delta_{1}$ implies that the third term is less than $\eta \lambda^{*} / 8$. Hence, for $\|f-g\|<\delta_{1}$ we have

$$
\left\|f_{1}-g_{1}\right\| \leqq 3 \eta \lambda^{*} / 8
$$

This implies that if $\delta=\min \left[\delta_{1}, \lambda^{*} \eta / 8\right]$ then, for all $f \in \Gamma_{0}$ and any $g$ with $\|f-g\|<\delta$, the set $U(f) \cap U(g)$ is not empty.

We now establish for each $f \in \Gamma_{0}$ that the closure $\bar{U}(f)$ of $U(f)$ is in the open ball $S(b, d)$. If $f \notin E$ this is obvious. Suppose $f \in E$ and let $u \in \bar{U}(f), p \in \partial S(b,\|b-f\|)$ be such that

$$
\|u-p\|=\min \|c-\bar{U}(f)\|
$$

with the minimum taken for $c \in \partial S(b,\|b-f\|)$. Then

$$
\begin{aligned}
\|u-p\| & \geqq\left\|f+\lambda^{*} t(f)-H(f)\right\|-\left\|u-f-\lambda^{*} t(f)\right\|-\|c-H(f)\| \\
& \geqq \eta \lambda^{*}[1 / 2-1 / 8-1 / 8]>0 .
\end{aligned}
$$


Since $f+\lambda^{*} t(f) \in S(b,\|b-f\|)$ it follows that $u \in S(b,\|b-f\|)$. Hence we have

$$
\bar{U}(f) \subset S(b, d) .
$$

Set

$$
\Gamma_{1}=\bigcup_{f \in \Gamma_{0}} \bar{U}(f)
$$

It is clear that $\Gamma_{1}$ is compact, $\Gamma_{1}$ contains $G$ and $\Gamma_{1} \subset S(b, d)$.

We show that it is also connected. Suppose, to the contrary, that there are nonempty sets $V_{1}, V_{2}$ open in $\Gamma_{1}$ so that

$$
\Gamma_{1}=V_{1} \cup V_{2}
$$

and $V_{1} \cap V_{2}$ is empty. Consider the mapping $M$ defined on $\Gamma_{0}$ by

$$
\begin{aligned}
M: f & =0, & \bar{U}(f) \subset V_{1}, \\
& =1, \quad & \bar{U}(f) \subset V_{2} .
\end{aligned}
$$

The mapping $M$ is well defined since $\bar{U}(f)$ is connected, i.e., $g \in \bar{U}(f), g \in V_{i}$ implies $\bar{U}(f) \subset V_{i}$. Further if $\bar{U}(f) \subset V_{i}$ and $\bar{U}(f) \cap \bar{U}(g)$ is not empty then $\bar{U}(g) \subset V_{i}$. If $\|f-g\|<\delta$ then $\bar{U}(f) \cap \bar{U}(g)$ is not empty and hence $M$ is continuous. Since $V_{1}$ and $V_{2}$ are not empty, the mapping $M$ is onto. Thus $M$ is a continuous mapping of $\Gamma_{0}$ onto $\{0,1\}$ which contradicts the connectedness of $\Gamma_{0}$. Thus $\Gamma_{1}$ is connected.

We have established that $\Gamma_{1}$ is a compact connected subset of $\mathscr{F}$ which contains $G$ and that

$$
\left\|b-\Gamma_{1}\right\|<\left\|b-\Gamma_{0}\right\| \text {. }
$$

This contradicts the definition of $\Gamma_{0}$ and establishes the lemma.

LEMMA 10. Let $\mathscr{F}$ be a smooth submanifold of a Minkowski space $B^{m}$ and consider $f \in \mathscr{F}, b \in B^{m}$ such that $l(b, f) \perp T(f)$. If $f$ is an accumulation point of points in $\mathscr{F} \cap \bar{S}(b,\|b-f\|)$, then the radius of curvature $\rho(f, r(f, b))$ of $\mathscr{F}$ at $f$ in the direction $r(f, b)$ satisfies

$$
\rho(f, r(f, b)) \leqq\|b-f\| .
$$

Proof. Consider a sequence $\left\{f_{i}\right\} \subset \mathscr{F} \cap \bar{S}(b,\|b-f\|)$ so that $f_{i} \rightarrow f$. Associate with each $f_{i}$ a point $b_{i} \in l$ so that $\left\|b_{i}-f\right\|=\left\|b_{i}-f_{i}\right\|$. Then

$$
f_{i} \in \partial S\left(b_{i},\left\|b_{i}-f_{i}\right\|\right) \subset \bar{S}(b,\|b-d\|) .
$$

Definition 7 implies immediately that

$$
\rho(f, r(f, b)) \leqq \operatorname{Lim}_{i \rightarrow \infty}\left\|b_{i}-f_{i}\right\| \leqq\|b-d\| .
$$

THEOREM 9. Let $\mathscr{F}$ be a closed submanifold of a smooth Minkowski space $B^{m}$ and assume for each compact subset $G$ of $\mathscr{F}$ there is a $K$ so that $\sigma(f) \leqq K \leqq \infty$ for $f \in G$. 
Then there is an open neighborhood $\mathscr{F}_{u}$ of $\mathscr{F}$ so that every point $b \in \mathscr{F}_{u}$ has a unique projection onto $\mathscr{F}$ i.e., $\boldsymbol{P}_{\mathscr{F}}(b)$ is single point.

Note that this does not imply that $\partial \mathscr{F}_{u}$ is bounded away from $\mathscr{F}$.

Proof. For $f \in \mathscr{F}$ we have from Lemma 7 that there is an $\varepsilon_{1}>0$ so that $S\left(f, \varepsilon_{1}\right) \cap \mathscr{F}$ is connected. Let $K$ be the bound on the curvature $\sigma(f)$ in $\bar{S}\left(f, \varepsilon_{1}\right) \cap \mathscr{F}$. Set

$$
\varepsilon=\min \left[1 / 3 K, \varepsilon_{1} / 2\right] .
$$

We show that if $b \in S(f, \varepsilon)$ and $b$ has two projections, $f_{1}$ and $f_{2}$, onto $\mathscr{F}$, then a contradiction is reached. It is clear that $f_{1}, f_{2} \in S\left(f, \varepsilon_{1}\right) \cap \mathscr{F}$. We have $S(b, 3 \varepsilon)$ $\supset S\left(f_{1}, \varepsilon\right)$ and hence $\bar{S}(b, 3 \varepsilon) \cap \mathscr{F}$ has a compact connected component $\Gamma_{1}$ which contains the compact set $f_{1} \cup f_{2}$. From Lemma 8 we have a compact connected subset $\Gamma_{0}$ of $\mathscr{F}$ so that

$$
\left\|b-\Gamma_{0}\right\|=\inf _{\Gamma}\|b-\Gamma\|=d,
$$

where the infimum is taken over all $\Gamma$ which contain $f_{1}$ and $f_{2}$.

If $\left\|b-\Gamma_{0}\right\|=\|b-\mathscr{F}\|$ then clearly $\Gamma_{0} \subset \partial S(b,\|b-\mathscr{F}\|)$, i.e., $\Gamma_{0}=\Gamma_{0}^{*}$ in the notation of Lemma 9. It follows from Theorem 3 that $l\left(b, f_{1}\right) \perp T\left(f_{1}\right)$ and we set $f^{*}=f_{1}$.

If $\|b-\mathscr{F}\|<\left\|b-\Gamma_{0}\right\|$ then Lemma 9 implies that there is a point $f^{*} \in \Gamma_{0}^{*}$ so that $l\left(b, f^{*}\right) \perp T\left(f^{*}\right)$. In either case $f^{*}$ is an accumulation point of points $f_{i} \in \bar{S}\left(b,\left\|b-\Gamma_{0}\right\|\right) \cap \mathscr{F}$. Lemma 10 implies that

$$
\rho\left(f^{*}, r\left(f^{*}, b\right)\right) \leqq\left\|b-\Gamma_{0}\right\| .
$$

We have then that

$$
1 / K \leqq\left\|b-\Gamma_{0}\right\| \leqq\left\|b-\Gamma_{1}\right\|<3 \varepsilon \leqq 1 / K .
$$

This is a contradiction and establishes that each $b \in S(f, \varepsilon)$ has a unique projection onto $\mathscr{F}$.

Set

$$
\mathscr{F}_{u}=\bigcup S(f, \varepsilon)
$$

for $f \in \mathscr{F}$, and the theorem is established.

Definition 9. The folding $\eta(f)$ of $\mathscr{F}$ at $f$ is $\eta(f)=\sup \{\rho \mid S(f, \lambda) \cap \mathscr{F}$ is connected for all $\lambda \leqq \rho\}$.

Lemma 7 states that a smooth submanifold of $B^{m}$ has positive folding at each point. The following is a quantititive restatement of Theorem 9.

Corollary 1. With $\mathscr{F}$ and $B^{m}$ as in Theorem 9 , let $b \in B^{m}$ and $f \in \boldsymbol{P}_{\mathscr{F}}(b)$ be given. Set

$$
\sigma=\max _{g}\{\sigma(g) \mid\|f-g\| \leqq \eta(f)\}, \quad \varepsilon=\min [1 / 3 \sigma, \eta(f) / 2] .
$$

If $\|b-\mathscr{F}\|<\varepsilon$ then $f$ is the unique projection of $b$ onto $\mathscr{F}$. 
It appears that $1 / 3 \sigma$ might be replaced by $1 / \sigma$, in which case this Corollary is obviously sharp. However, the method of proof of Theorem 9 does not allow this. A related result with a stronger hypothesis is given in the next section.

Another consequence of the proof of Theorem 9 is

Corollary 2. With $\mathscr{F}$ and $B^{m}$ as in Theorem 9, suppose that a connected component of $S(b, d) \cap \mathscr{F}$ contains $f_{1}, f_{2} \in \boldsymbol{P}_{\mathscr{F}}(b)$. Then $\mathscr{F}$ has curvature of at least $1 / 3 d$ in $\bar{S}(b, d) \cap \mathscr{F}$.

\section{On the topological structure of $\operatorname{Nup}(\mathscr{F})$.}

Definition 10. A point $b \in B^{m}$ is a Nup (nonunique projection) point of $\mathscr{F}$ if $\boldsymbol{P}_{\mathscr{F}}(b)$ consists of more than one point. Further

$$
\operatorname{Nup}(\mathscr{F})=\overline{\{b \mid b \text { is a nup point of } \mathscr{F}\}}
$$

where the bar indicates that the closure is taken.

In a study of the uniqueness of projections onto $\mathscr{F}$ we are naturally interested in the nature of Nup $(\mathscr{F})$. In this section we exhibit a direct relationship between the topological properties of $\mathscr{F}$ and $\operatorname{Nup}(\mathscr{F})$. In order to establish this we perform the usual [2] one point compactification of $B^{m}$ to obtain the $m$-sphere $S^{m}$ (which is to be distinguished from a sphere in $B^{m}$ ). The additional ideal point is assigned to $\operatorname{Nup}(\mathscr{F})$.

The closure operation may add a certain number of points to Nup $(\mathscr{F})$ which are not nup points. These are rather special points which one would intuitively expect to be "centers of curvature." Conditions under which this is true are given in the next theorem.

THEOREM 10. Let $\mathscr{F}$ be a submanifold with continuous curvature of a Minkowski space $B^{m}$. Suppose that $b \in \operatorname{Nup}(\mathscr{F})$ and $b$ is not $a$ nup point and $f=\boldsymbol{P}_{\mathscr{F}}(b)$. Then

$$
\|b-\mathscr{F}\|=\rho(f, r(f, b)) \text {. }
$$

Proof. For compactness of notation set

$$
r^{*}=r(f, b), \quad \rho^{*}=\rho\left(f, r^{*}\right) .
$$

It follows from Theorem 7 that $\|b-\mathscr{F}\| \leqq \rho^{*}$. The assumption that $\|b-\mathscr{F}\|$ $=\rho^{*}-\eta, \eta>0$, leads to a contradiction as follows. Since the curvature is continuous at $f$, there is an $\varepsilon>0$ so that $\|f-g\| \leqq \varepsilon,\left\|r^{*}-r(g, c)\right\|<\varepsilon$ implies that $\rho(g, r(g, c))$ $\geqq \rho^{*}-\eta / 2$. It is known (and easily shown) that $\boldsymbol{P}$ is continuous at $b$ in the sense that given $\varepsilon>0$ there is a $\delta>0$ so that $\|b-c\|<\delta$ implies $\|f-P(c)\|<\varepsilon$. Thus there is a $\delta>0$ so that $\|b-c\|<\delta$ implies that $\|f-\boldsymbol{P}(c)\|<\varepsilon$ and $\left\|r^{*}-r(\boldsymbol{P}(c), c)\right\|<\varepsilon$. Hence every point $c$ in $S(b, \delta)$ has $\boldsymbol{P}_{\mathscr{F}}(g) \subset S(f, \varepsilon) \cap \mathscr{F}$ and $\rho(g, r(g, c)) \geqq \rho^{*}-\eta / 2$. Since $B^{m}$ is Minkowskian, every point $h$ on $r(g, c)$ with $\|h-\mathscr{F}\| \leqq \rho^{*}-3 \eta / 4$ has a unique projection onto $\mathscr{F}$. Set $\mu=\min [\delta, \eta / 4]$ and this implies that

$$
S(b, \mu) \cap \operatorname{Nup}(\mathscr{F})
$$

is empty. This is a contradiction and completes the proof. 
THEOREM 11. Let $\mathscr{F}$ be a closed submanifold with continuous curvature of a Minkowski space $B^{m}$. Then $\operatorname{Nup}(\mathscr{F})$ is a deformation retract of $S^{m}-\mathscr{F}$.

Proof. For $b \notin \mathscr{F}, b \notin \operatorname{Nup}(\mathscr{F})$ set $f=\boldsymbol{P}(b), r^{*}=r(f, b)$,

$$
\begin{aligned}
\alpha^{\prime}(b) & =\min \left\{\|f-c\| \mid c \in r^{*} \cap \operatorname{Nup}(\mathscr{F})\right\}, \\
\alpha(b) & =\alpha^{\prime}(b) /\|f-b\| .
\end{aligned}
$$

We take the minimum of the empty set to be $+\infty$ and note that $\alpha(b)>1$. Define $\phi(t, b)=b$ for $b \in \operatorname{Nup}(\mathscr{F})$ and

$$
\phi(t, b)=\boldsymbol{P}(b)+\frac{b-\boldsymbol{P}(b)}{1-t[1-1 / \alpha(b)]}, \quad b \notin \operatorname{Nup}(\mathscr{F}) .
$$

It is clear that $\phi(0, b)=b$ and $\phi(1, b) \in \operatorname{Nup}(\mathscr{F})$. For $b \notin \operatorname{Nup}(\mathscr{F}), \boldsymbol{P}(b)$ is a continuous function of $b$. Further $\phi(t, b)$ is continuous in $t$ and $\alpha$ for $0 \leqq t \leqq 1$ and $\alpha>1$ in the topology of $S^{m}$. We show that $\alpha(b)$ is a continuous function of $b$. It follows directly from Theorem 7 that

$$
\alpha(b) \leqq \rho\left(f, r^{*}\right) .
$$

The assumption that there is a sequence $\left\{c_{i}\right\}$ which converges to $b \notin \operatorname{Nup}(\mathscr{F})$ so that $\left|\alpha\left(c_{i}\right)-\alpha(b)\right| \geqq \eta>0$ leads to a contradiction as follows. If $\alpha\left(c_{i}\right)<\alpha(b)$ for infinitely many $i$ then a subsequence of $\left\{\phi\left(1, c_{i}\right)\right\}$ converges to a point

$$
c \in \operatorname{Nup}(\mathscr{F}) \cap r^{*} \text {. }
$$

Thus $\|f-c\|<\|f-\phi(1, b)\|=\alpha^{\prime}(b)$ which contradicts the definition of $\alpha^{\prime}(b)$.

Suppose that $\alpha(b)<\alpha\left(c_{i}\right)$ for infinitely many $i$ then $\phi(1, b)$ is contained in the interior of the segment $s(f, c)$. Since $B^{m}$ is Minkowskian it follows from Lemma 1 that $\phi(1, b)$ is not a nup point. Since $\phi(1, b) \in \operatorname{Nup}(\mathscr{F})$ it follows from Theorem 10 that

$$
\|\phi(1, b)-\mathscr{F}\|=\rho\left(f, r^{*}\right)=\rho^{*} .
$$

Since the curvature is continuous, given $\eta / 2>0$ there is a $\delta>0$ so that $\|f-g\|<\delta$ implies that $\rho(g, r(g, c))<\rho^{*}+\eta / 2$. Thus for $\left\|c_{i}-b\right\|$ sufficiently small

$$
\alpha^{\prime}\left(c_{i}\right) \leqq \rho\left(\boldsymbol{P}\left(c_{i}\right), r\left(\boldsymbol{P}\left(c_{i}\right), c_{i}\right)\right)<\rho^{*}+\varepsilon=\alpha^{\prime}(b)+\eta / 2
$$

which contradicts the supposition on $\alpha\left(c_{i}\right)$.

If $b \in \operatorname{Nup}(\mathscr{F})$ and $b$ is not a nup point then the preceding argument shows that $\alpha(b)$ is continuous.

Suppose that $b$ is a nup point. For points $b \in \operatorname{Nup}(\mathscr{F})$ then the continuity of $\phi(t, b)$ is clear. Further if $c_{i} \rightarrow b$ and $\alpha\left(c_{i}\right)<1-\eta$, then a contradiction is reached as before. Likewise if $\|b-\mathscr{F}\|+\eta<\alpha^{\prime}\left(c_{i}\right) \leqq K<\infty$ a preceding argument leads to a contradiction.

Finally we show that if $\alpha(b)<\infty$, then $\left|\alpha\left(c_{i}\right)-\alpha(b)\right|$ cannot be unbounded. If $\phi(1, b)$ is not a nup point, then the above argument applies. The remaining case is 
based on the following fact: Let $\partial S_{1}$ and $\partial S_{2}$ be two closed spheres in a Minkowski space $B^{m}$ which have a common hyperplane of support at a common point which is collinear with the centers of the spheres. Then either $\partial \bar{S}_{1}=\partial \bar{S}_{2}$ or $\partial \bar{S}_{1} \cap \partial \bar{S}_{2}$ consists of a single point. Let $z$ be a point on the ray $r(f, b)$ with $\|z-\mathscr{F}\|$ $=2\|\phi(1, b)-\mathscr{F}\|$. Then $S(z,\|z-f\|)$ contains points of $\mathscr{F}$. Furthermore, as $c_{\mathfrak{i}} \rightarrow b$, there are points $z_{i} \in r\left(\boldsymbol{P}\left(c_{i}\right), c_{i}\right)$ which converge to $z$ and if $\left|\alpha\left(c_{i}\right)\right| \rightarrow+\infty$, these points have a unique projection onto $\mathscr{F}$ near $f$. However $S\left(z_{i},\left\|z_{i}-\mathscr{F}\right\|\right)$ is arbitrarily close to $S(z,\|z-f\|)$ and hence for $i$ sufficiently large $S\left(z_{i},\left\|z_{i}-\mathscr{F}\right\|\right)$ contains points of $\mathscr{F}$ bounded away from $f$. This is a contradiction and concludes the proof.

Examples show that the hypothesis of continuous curvature is essential to the continuity of $\phi(t, b)$. However, these examples also suggest that the conclusion of this theorem is valid with a weaker hypothesis on $\mathscr{F}$. This theorem is stated in [8], but the hypothesis of the continuity of the curvature is missing.

One may combine this theorem and the Alexander duality theorem to establish a precise relationship between the topological nature of $\mathscr{F}$ and $\operatorname{Nup}(\mathscr{F})$. Let $R_{\pi}^{p}(\mathscr{X})$ denote the $p$ th Betti number of $\mathscr{X}$ for chains mod $\pi[4]$. We have

COROLlaRY 1. With the assumptions of Theorem 11 and that the Alexander duality theorem applies, then in $S^{m}$ we have the following relation

$$
R_{\pi}^{p}(\mathscr{F})=R_{\pi}^{p}(\operatorname{Nup}(\mathscr{F}))+\delta_{p, m-1}-\delta_{p, 0}
$$

where $\delta_{i j}$ is the Kronecker $\delta$.

This corollary may be used to establish a variety of more specialized results. The following typical result is a consequence of Theorem 11 and known facts on Motzkin sets. See [10, Theorems 7.8, 7.10].

COROLlaRY 2. The only submanifolds $\mathscr{F}$ of a smooth Minkowski space for which Nup $(\mathscr{F})$ consists of exactly p points is a convex body with exactly $p$ disjoint balls removed.

7. A related concept and nonmanifold problems. The study of projections onto submanifolds is a natural step toward a geometric theory of nonlinear approximation. However, such a theory does not apply to many of the most interesting nonlinear approximation problems. For example, let $F(A, x)$ be a varisolvent function (see [7] for definitions), $X=\left(x_{1}, \ldots, x_{m}\right)$ and define

$$
\mathscr{F}=\left\{\left(F\left(A, x_{1}\right), F\left(A, x_{2}\right), \ldots, F\left(A, x_{m}\right)\right) \mid \text { all } A\right\}
$$

as a subset of $m$-dimensional vector space $B^{m}$. Varisolvent functions are among the most interesting nonlinear approximating functions. The following theorem consolidates known facts and results whose proofs will appear in [9].

TheOREM 12. Assume the degree $n(A)$ of $F(A, x)$ is not constant. Then $\mathscr{F}$ is neither convex nor a manifold nor closed. 
Federer introduced the concept of reach in [3] in order to give a unified treatment of projections onto both manifolds and convex sets in Euclidean space. We make a modified definition of reach which extends the concept to Banach spaces and makes the reach a local property (analogous to curvature) of $\mathscr{F}$.

DeFINITION 11. The reach of $\mathscr{F}$ at $f$ is

$$
\omega(f)=\operatorname{Lim}_{\varepsilon \rightarrow 0} \min _{b}\{\|f-b\| \mid b \in \operatorname{Nup}[\mathscr{F} \cap S(f, \varepsilon)]\} .
$$

The reach $\omega(\mathscr{F})$ of $\mathscr{F}$ is inf $\omega(f)$ for $f \in \mathscr{F}$.

A variety of properties of the reach are given in [3]. Some, but not all, of these carry over directly to the present context.

In this paper the study of the uniqueness of projections has been made using a concept of curvature analogous to that of classical geometry. It is possible that projections and uniqueness are more natural and intrinsic concepts than curvature (i.e., perhaps one should define curvature in terms of the uniqueness of projections). When the more general and interesting problems are studied it seems certain that something similar to reach will play the role of curvature and enter the theory in an essential way.

\section{REFERENCES}

1. H. Buseman, The geometry of geodesics, Academic Press, New York, 1955.

2. J. Dugundji, Topology, Allyn and Bacon, Boston, 1966.

3. H. Federer, Curvature measures, Trans. Amer. Math. Soc. 93 (1959), 418-491.

4. S. Lefschetz, Introduction to topology, Princeton Univ. Pesss, Princeton, N. J., 1949.

5. J. W. Milnor, Morse theory, Princeton Univ. Press, Princeton, N. J., 1963.

6. T. S. Motzkin, Approximation by curves of a unisolvent family, Bull. Amer. Math. Soc. 55 (1949), 789-793.

7. J. R. Rice, On the existence and characterization of best nonlinear Tchebycheff approximations, Trans. Amer. Math. Soc. 110 (1964), 88-97.

8. - " "Nonlinear approximation," in Approximation of functions, pp. 111-133, (H. L. Garabedian, Editor), Elsevier, Amsterdam, 1965.

9. — The approximation of functions, Vol. II, Addison-Wesley, Reading, Mass. (to appear).

10. F. A. Valentine, Convex sets, McGraw-Hill, New York, 1964.

Purdue University,

LAFAYETTE, INDIANA 